\title{
Die Beziehungen Schweiz - EU als Modell für die Gestaltung des Verhältnisses Großbritanniens zur EU?
}

\author{
Astrid Epiney
}

Dieser Beitrag wurde erstmals wie folgt veröffentlicht:

Astrid Epiney, Die Beziehungen Schweiz - EU als Modell für die Gestaltung des Verhältnisses Großbritanniens zur EU?, in: Malte Kramme/Christian Baldus/Martin Schmidt-Kessel (Hrsg.), Brexit und die juristischen Folgen, Baden-Baden 2017, 77-100. Es ist möglich, dass diese publizierte Version - die allein zitierfähig ist - im Verhältnis zu diesem Manuskript geringfügige Modifikationen enthält.

\section{Einleitung}

Zwar ist das Ergebnis der sog. Brexit-Abstimmung in Großbritannien (im Gegensatz z.B. zu denjenigen von Volkabstimmungen in der Schweiz) für die Regierung und insbesondere das Parlament nicht rechtlich verbindlich. Nichtsdestotrotz ist zu erwarten, dass Großbritannien in näherer Zukunft dem Europäischen Rat gemäß Art. 50 Abs. 2 EUV seine Absicht, aus der Union auszutreten, mitteilt. Damit wird sich die Frage nach der zukünftigen Gestaltung des Verhältnisses des Vereinigten Königreichs zur Union stellen, ein Aspekt, der auch durch das grundsätzlich abzuschließende „Austrittsabkommen“ berücksichtigt werden soll, ohne dass dem Vertrag hier jedoch nähere Präzisierungen zu entnehmen wären. Es steht jedoch außer Frage, dass im Zuge der Durchführung des Austritts Großbritanniens nicht nur diverse Fragen, die sich aufgrund der „Loslösung“ zwangsläufig ergeben (wie z.B. diejenige nach den erworbenen Rechten der Unionsbürger), zu beantworten sind, sondern auch die grundsätzliche „Architektur“ der Beziehungen Großbritanniens zur Union zu gestalten sein wird, wobei beide Aspekte übrigens - je nach gewählter Lösung - in einem mehr oder weniger engen Zusammenhang stehen.

Für die somit neu zu gestaltenden Beziehungen des Drittstaats Großbritannien (so der Austritt vollzogen wird) zur Union sind selbstredend verschiedene Modelle denkbar, dies auch und gerade in Anknüpfung an die diversen völkerrechtlichen Abkommen, welche die Union bereits mit Drittstaaten, die an der Partizipation an Teilen des unionsrechtlichen Besitzstands, insbesondere im Bereich des Binnenmarkts, interessiert sind, abgeschlossen hat. Ein mögliches „Vorbild“ in diesem Zusammenhang könnten die Beziehungen Schweiz - EU sein, dies auch vor dem Hintergrund, dass die (wirtschaftlich leistungsfähige) Schweiz schon aufgrund ihrer geographischen Lage und der (wohl auch damit einhergehenden) ausgesprochen engen wirtschaftlichen Verflechtung mit der Union ein sehr großes Interesse an einer Einbindung insbesondere in Teile des Binnenmarktes, aber auch in sonstige Aspekte des unionsrechtlichen Besitzstands hat, wobei dieses Interesse durchaus auch gegenseitig ist. Diese Ausgangslage dürfte sich in mancher Beziehung für Großbritannien ähnlich präsentierten. 
Vor diesem Hintergrund sei im Folgenden danach gefragt, ob den Beziehungen Schweiz - EU für die zukünftige Gestaltung des Verhältnisses Großbritanniens zur Union eine Art Leitbildfunktion zukommen könnte, dies unter Einbezug der sich hier stellenden (offenen) Fragen. Hierzu sollen in einem ersten Schritt (II.) die wesentlichen Charakteristika der bestehenden (sog. Bilateralen) Abkommen der Schweiz mit der Union skizziert werden, um sodann auf die sich derzeit stellenden offenen Fragen hinzuweisen (III.). Auf dieser Grundlage können in einem abschließenden Fazit (IV.) einige Gedanken zu den Eckwerten von Abkommen der Union mit Großbritannien nach dem „Vorbild“ der Bilateralen Abkommen Schweiz - EU und damit zur „Übertragbarkeit“ des „Bilateralen Weges“, den die Schweiz im Verhältnis zur Union geht, auf das zukünftige Verhältnis Großbritanniens zur Union formuliert werden.

\section{Zu den Charakteristika der „Bilateralen Abkommen“ - eine Skizze}

Seit der Ablehnung des EWR-Beitritts durch Volk und Stände am 6. Dezember 1992 beruhen die Beziehungen der Schweiz zur Europäischen Union auf dem sog. bilateralen Ansatz, bilateral insofern, als auf der einen Seite die Schweiz, auf der anderen die EU und (teilweise) ihre Mitgliedstaaten stehen, dies im Gegensatz zum EWR, der die Beziehungen einer (immer kleiner gewordenen) Staatengruppe (der EFTA-Staaten, außer der Schweiz) zur EU regelt. Inzwischen gibt es zwei „Pakete“ sog. Bilateraler Abkommen, ${ }^{1}$ die sektoriell eine Reihe von Bereichen regeln und in der Regel eine Einbindung der Schweiz in den Unionsbesitzstand vorsehen; ${ }^{2}$ hinzukommen zahlreiche weitere Verträge, in jüngerer Zeit z.B. das sog. Zollsicherheitsübereinkommen ${ }^{3}$, das „Europol-Abkommen“ (2004) ${ }^{4}$ oder das Wettbewerbsabkommen $(2013)^{5}$, in der Vergangenheit etwa das Freihandelsabkommen aus

1 Der Ausdruck „Bilaterale Abkommen“ ist im Gegensatz zu dem als multilateral angesehenen Ansatz insbesondere des EWR zu sehen. Aus rechtlicher Sicht ist er jedoch zumindest ungenau, da die Abkommen teilweise sog. gemischte Abkommen sind (und somit neben der Union auch die Mitgliedstaaten Vertragsparteien sind), so dass es insofern um multilaterale Abkommen geht). Vor diesem Hintergrund trifft der Ausdruck „sektorielle Abkommen“ die Rechtslage eigentlich besser, da er Bezug auf die bereichsspezifische Regelung der verschiedenen Dossiers nimmt. Gleichwohl werden im Folgenden die inzwischen gebräuchlichen Ausdrücke „Bilaterale I“ und „Bilaterale II“ verwandt.

2 Vgl. zu den Bilateralen Verträgen ausführlich und m.w.N. Astrid Epiney/Beate Metz/Benedikt Pirker, Zur Parallelität der Rechtsentwicklung in der EU und in der Schweiz. Ein Beitrag zur rechtlichen Tragweite der „Bilateralen Abkommen“, 2012, 95 ff.; Thomas Cottier/Nicolas Diebold/Isabel Kölliker/Rachel Liechti McKee/Matthias Oesch/Tetyana Payosova/Daniel Wüger, Die Rechtsbeziehungen der Schweiz und der Europäischen Union, 2014, passim.

3 Abkommen über die Erleichterung der Kontrollen und Formalitäten im Güterverkehr und über zollrechtliche Sicherheitsmaßnahmen (,24-Stunden-Regel“), SR 0.631.242.05.

4 Abkommen zwischen der Schweiz und dem Europäischen Polizeiamt, SR 0.362.2.

5 Abkommen zwischen der Schweiz und der EU über die Zusammenarbeit bei der Anwendung ihrer Wettbewerbsrechte, SR 0.251.268.1. 
dem Jahr $1972^{6}$ oder das sog. Versicherungsabkommen aus dem Jahr 19897. Darüber hinaus sind auch sonstige multilaterale völkerrechtliche Abkommen, an denen nicht nur die Schweiz und die EU sowie ggf. ihre Mitgliedstaaten, sondern noch weitere Staaten als Vertragsparteien beteiligt sind, auch für die Beziehungen Schweiz - EU von Bedeutung und zu beachten. Für die wirtschaftlichen Beziehungen spielt hier zweifellos das WTO-Vertragswerk eine besondere Rolle.

Insgesamt kommt jedoch sowohl aus rechtlicher als auch aus wirtschaftlicher und politischer Sicht den beiden Paketen Bilateraler Abkommen zweifellos eine besondere Bedeutung zu, so dass sich die folgenden Ausführungen auf diese beschränken: Nach einem kurzen Überblick über ihre wesentlichen Charakteristika (1.) sollen sodann die in unserem Zusammenhang besonders interessierenden institutionellen Fragen skizziert werden (2.).

\section{1. $\quad$ Überblick}

Das erste „Paket“ der sog. bilateralen und sektoriellen Abkommen zwischen der EU bzw. ihren Mitgliedstaaten einerseits und der Schweiz andererseits wurde am 21. Juni 1999 unterzeichnet und trat am 1. Juni $2002^{8}$ in Kraft. Es betrifft folgende Bereiche: Personenfreizügigkeit, technische Handelshemmnisse, landwirtschaftliche Produkte, Landverkehr, Luftverkehr und öffentliches Auftragswesen.

Bald nach Abschluss der sog. „Bilateralen I“ wurden die Verhandlungen zu den sog. „Bilateralen II“ aufgenommen, und im Oktober 2004 wurde dieses zweite Paket unterzeichnet, und die einzelnen Abkommen traten danach (abgesehen vom noch nicht in Kraft getretenen, aber vorläufig angewendeten Betrugsabkommen) zu unterschiedlichen Zeitpunkten im Zuge der Ratifikation in Kraft. ${ }^{9}$ Inhaltlich befassen sich die Bilateralen II einerseits mit den „Restthemen“ („left overs“) der Bilateralen I, andererseits betreffen sie neue politische Anliegen beider Seiten. Auf dieser Grundlage umfasst das Paket der „Bilateralen II“ - nach der Zurückstellung des Dienstleistungsdossiers - folgende Bereiche: Besteuerung der Ruhegehälter von in der Schweiz ansässigen EU-Beamten, verarbeitete Landwirtschaftsprodukte, Teilnahme der Schweiz an der Europäischen Umweltagentur, Zusammenarbeit im Bereich der Statistik, Teilnahme der Schweiz an verschiedenen Programmen in den Bereichen Bildung, Berufsbildung und Jugend, Teilnahme an „Dublin“ und am „Schengen-acquis“, Zinsbesteuerung und Betrugsbekämpfung.

SR 0.632.401

Abkommen zwischen der Schweiz und der EWG betreffend die Direktversicherung mit Ausnahme der Lebensversicherung, SR 0.961.1

$8 \quad$ Für die Texte aller Abkommen s. BB1 1999, 6489 ff.; ABl 2002 L 114, 1 ff. Aus der Literatur zu diesen Abkommen die Nachweise in Fn. 2.

$9 \quad$ Text und Botschaft zu den Bilateralen II in BB1. 2004, 5965 ff. 
Die Bilateralen Abkommen weisen trotz aller Unterschiede sowohl in materieller als auch in institutioneller Hinsicht eine Reihe von Gemeinsamkeiten auf, wobei diese aber auch teilweise zwischen den Bilateralen I und den Bilateralen II differieren und mitunter bei einzelnen Abkommen auch Besonderheiten zu verzeichnen sind.

Hintergrund und Ausgangspunkt ist in diesem Zusammenhang die formale Ausgestaltung der Bilateralen Abkommen als „klassische“ völkerrechtliche Verträge. Insofern handelt es sich grundsätzlich nicht um eigentliche Integrationsverträge in dem Sinn einer echten Einbindung in die Integration der EU-Staaten bzw. die EU. Dieser grundsätzliche Ansatz hat sich sowohl in der inhaltlichen Ausgestaltung der Abkommen (die auf den Prinzipien der Gleichwertigkeit der Gesetzgebung bzw. Standards beruhen und gerade keine eigentliche Integration in den unionsrechtlichen Besitzstand vorsehen) als auch in der institutionellen Struktur (die „klassisch völkerrechtlich“" ausgestaltet ist) niedergeschlagen.

Eine Ausnahme stellt hier das Luftverkehrsabkommen aus den Bilateralen I dar, das in verschiedener Hinsicht eine ,echte“ Integration in den unionsrechtlichen Besitzstand vorsieht, insbesondere in Bezug auf die Befugnisse der Organe der Union. Auch das Schengen/DublinAbkommen kann vor dem Hintergrund der sehr weitgehenden Verpflichtungen der Schweiz zur Übernahme des einschlägigen unionsrechtlichen Besitzstands und seiner Weiterentwicklungen als Teilintegrationsvertrag angesehen werden.

Die wesentlichen Charakteristika der Bilateralen Abkommen können auf dieser Grundlage und vor dem Hintergrund der Zielsetzung des vorliegenden Beitrags wie folgt zusammengefasst werden: ${ }^{10}$

- Die einzelnen Abkommen der beiden Pakete Bilateraler Abkommen stellen jeweils getrennte, in sich geschlossene Verträge dar. So sind auch grundsätzlich bei allen Abkommen relevante Fragen, wie etwa Streitbeilegung oder institutionelle Ausgestaltung, jeweils in den verschiedenen Abkommen für jeden Bereich geregelt.

- Gleichzeitig sind die Abkommen aber auch miteinander verbunden, wobei diese Verbindung bei den Bilateralen II (nur) politischer Natur ist, die Bilateralen I hingegen auch rechtlich miteinander verknüpft sind: Die sieben Abkommen der Bilateralen I stellen insofern ein „Paket“ dar, als die Abkommen - wie in den Schlussbestimmungen aller Abkommen niedergelegt - nur zusammen in Kraft treten konnten, und die Nichtverlängerung oder Kündigung eines der Abkommen dazu führt, dass auch alle anderen Abkommen außer Kraft treten bzw. nicht mehr angewandt werden (sog. Guillotine-Klausel).

- Der materielle Inhalt der Abkommen ist grundsätzlich statisch ausgestaltet, woran auch die häufig anzutreffende Anknüpfung an den unionsrechtlichen Besitzstand sowie die regelmäßig vorgesehene Möglichkeit der Weiterentwicklung - insbesondere im Hinblick

10 Vgl. insoweit auch schon Epiney/Metz/Pirker, Parallelität der Rechtsentwicklung in der EU und in der Schweiz (Fn. 2), 96 ff., worauf die folgenden Ausführungen zurückgreifen. 
auf die Anpassung der Abkommensinhalte an die Entwicklung des EU-Rechts - in struktureller Sicht wenig ändern, wobei hier jedoch beim Luftverkehrsabkommen und der Schengen / Dublin-Assoziierung gewisse Relativierungen zu verzeichnen sind.

- $\quad$ Im Übrigen erfassen die verschiedenen Abkommen jeweils nur bestimmte, im Verhältnis etwa zum EWR relativ beschränkte Bereiche, so dass von einem sektorspezifischen Vorgehen gesprochen werden kann und es gerade nicht (wie beim EWR) um eine umfassende Teilnahme am Binnenmarkt geht, wenn die Gesamtheit der Abkommen auch $\mathrm{zu}$ einer sehr weitgehenden Einbindung der Schweiz in den unionsrechtlichen Besitzstand, insbesondere auch den Binnenmarktbesitzstand, führt.

- In Bezug auf die inhaltliche Ausrichtung der Abkommen kann zwischen Kooperationsabkommen, die die Zusammenarbeit in bestimmten Bereichen oder die Teilhabe der Schweiz an bestimmten EU-Programmen betreffen, Liberalisierungs- und Harmonisierungsabkommen (z.B. in den Bereichen Personen- und Landverkehr sowie bei anderen die Teilnahme am Binnenmarkt betreffenden Abkommen) sowie (Teil-) Integrationsverträgen (wie beim Luftverkehrsabkommen, aber wohl auch beim Schengen/Dublin-Abkommen) unterschieden werden.

- Aufgrund des völkerrechtlichen Charakters der Bilateralen Abkommen obliegen Anwendung und Auslegung der Abkommen grundsätzlich jeweils den zuständigen Behörden und Gerichten der Vertragsparteien, wenn auch einzelne Abkommen hier weitergehende Vorgaben vorsehen. ${ }^{11}$

- $\quad$ Vor dem Hintergrund, dass zahlreiche Abkommen Mechanismen der Weiterentwicklung der Abkommen im Hinblick auf die Anpassung ihres materiellen Inhalts an die Rechtsentwicklungen in der EU vorsehen, räumen die Abkommen der Schweiz häufig gewisse Beteiligungsrechte im Rahmen des Rechtsetzungsprozesses innerhalb der EU ein, dies soweit es um Rechtsakte geht, die Eingang in das entsprechende Abkommen finden (können).

- Schließlich ist auf die institutionelle Struktur der Abkommen hinzuweisen: Die Abkommen sehen grundsätzlich ${ }^{12}$ jeweils einen Gemischten Ausschuss vor, der aus Vertretern der Union einerseits und der Schweiz andererseits zusammengesetzt ist und einstimmig entscheidet. Die Kompetenzen dieser Gemischten Ausschüsse betreffen im Wesentlichen drei große Bereiche:

- Informationsaustausch, Konsultationen und sonstige „weiche“ Mechanismen im Hinblick auf die Sicherstellung des guten Funktionierens der Abkommen;

11 Hierzu, m.w.N., Epiney/Metz/Pirker, Parallelität der Rechtsentwicklung in der EU und in der Schweiz (Fn. 2), $99 \mathrm{f}$.

12 Ausnahmen in dem Sinn, dass ein Abkommen keinen Gemischten Ausschuss vorsieht, sind im Zinsbesteuerungs- und Ruhegehälterabkommen sowie im Bildungsabkommen zu verzeichnen. Das Agrarabkommen verfügt über zwei Gemischte Ausschüsse. 
- $\quad$ Streitbeilegung, wobei hier keine für die Parteien verbindlichen Beschlüsse gefasst werden können;

- $\quad$ Fassung verbindlicher Beschlüsse, soweit dies ausdrücklich vorgesehen ist, wobei in erster Linie die Befugnisse zur Anpassung der Verweise der Sekundärrecht enthaltenden Anhänge sowie der Beschluss von Schutzmaßnahmen von Bedeutung sind.

\section{Insbesondere: zu einigen zentralen institutionellen Aspekten ${ }^{13}$}

Die genaue rechtliche Tragweite völkerrechtlicher Abkommen hängt nicht nur von den erfassten materiellen Bereichen, sondern auch und gerade der Art und Weise ihrer institutionellen Ausgestaltung ab. Hier weisen denn auch die Bilateralen Abkommen einige Besonderheiten im Verhältnis zu „klassischen“ völkerrechtlichen Verträgen auf. Von besonderer Bedeutung ist dabei die „Anbindung“ an den unionsrechtlichen Besitzstand, wobei zwischen dem bestehenden Besitzstand (a) und seiner Weiterentwicklung (b) unterschieden werden kann. Relevant sind in diesem Zusammenhang auch die Auslegung der Abkommen (c) sowie Rechtsschutzaspekte (d).

a) Zur Reichweite der „Anlehnung“ an den unionsrechtlichen Besitzstand

Die Frage nach der Reichweite der „Übernahme“ von Teilen des unionsrechtlichen Besitzstandes in ein (Bilaterales) Abkommen zwischen der Schweiz und der EU ist auf der Grundlage des Gegenstands bzw. des materiellen Anwendungsbereichs eines Abkommens zu sehen: In all denjenigen Fällen, in denen ein Abkommen die „Integration“ der Schweiz in einen Teil des Unionsrechts anstrebt, erfolgt in der Regel eine relativ weitgehende Anlehnung an das bereits bestehende Unionsrecht. Dessen Umfang wird einerseits durch den eigentlichen Regelungszweck bzw. -schwerpunkt des jeweiligen Abkommens, andererseits durch mit diesen in enger Verbindung stehenden Bereichen bestimmt.

Diese „Übernahme“ unionsrechtlicher Bestimmungen in Bilateralen Abkommen mit der Schweiz erfolgt in den derzeit bestehenden Abkommen entweder durch einen direkten Verweis auf unionsrechtliche $\mathrm{Akte}^{14}$ oder aber durch eine Anlehnung der Formulierung

13 Vgl. insoweit bereits Astrid Epiney, Beziehungen Schweiz - EU: Status quo und Perspektiven, in: Patrik Schellenbauer/Gerhard Schwarz (Hrsg.), Bilateralismus - was sonst? Eigenständigkeit trotz Abhängigkeit, Zürich 2015, 25 (43 ff.), worauf der folgende Abschnitt teilweise beruht.

14 Indem in Anhängen zu einem Abkommen Sekundärrechtsakte aufgeführt werden, die die Schweiz im Ergebnis anzuwenden hat (,gleichwertige Rechtsetzung“). S. z.B. Anhang II, III Personenfreizügigkeitsabkommen oder Anhang I Landverkehrsabkommen. 
abkommensrechtlicher Bestimmungen an unionsrechtliche Regelungen. ${ }^{15}$ Abgesehen von derartigen, sich vom Unionsrecht inspirierenden abkommensrechtlichen Bestimmungen enthalten die Bilateralen Abkommen aber auch (wobei dies jedenfalls in den politisch und rechtlich bedeutenden Bereichen die Ausnahme darstellt),,autonome“ Bestimmungen, also solche, die sich nicht in irgendeiner Form an den unionsrechtlichen Besitzstand anlehnen.

Welche Inhalte nun genau ein Abkommen hat, welche der angesprochenen Methoden gewählt wird und damit inwieweit eine Anlehnung an den unionsrechtlichen Besitzstand erfolgt, hängt natürlich vom genauen Inhalt und den Zielsetzungen, die mit einem Abkommen verfolgt werden, ab. Festzuhalten bleibt aber jedenfalls, dass in denjenigen Fällen, in denen es um eine teilweise Einbindung der Schweiz in gewisse Unionspolitiken bzw. um Bereiche, die bereits durch das EU-Recht geregelt sind, geht, aus politischer Sicht in aller Regel lediglich die auf die eine oder andere Art zu bewerkstelligende (teilweise oder ganze) Übernahme des einschlägigen unionsrechtlichen Besitzstands in Betracht kommt. Aber auch die Zielsetzungen der Abkommen dürften häufig nur über eine solche „Ausdehnung“ des unionsrechtlichen Besitzstandes erreicht werden können, so insbesondere bei denjenigen Abkommen, bei denen es um eine Teilnahme der Schweiz am Binnenmarkt geht.

In Bezug auf die Form der Übernahme des Unionsrechts in den Abkommen dürfte jedenfalls das Interesse der EU dahin gehen, verstärkt mit direkten Bezugnahmen auf bestehendes Unionsrecht zu arbeiten, kann doch auf diese Weise eine möglichst weitgehende Übernahme der unionsrechtlichen Vorgaben erreicht werden und zudem die Weiterentwicklung der Abkommen erleichtert werden. Es ist denn auch bezeichnend, dass gerade in der in dieser Beziehung weitgehenden Schengen-Assoziierung - die von der Europäischen Union als eine Art Mindeststandard für weitere Abkommen angesehen wird ${ }^{16}$ - ausschließlich mit dieser Technik gearbeitet wird.

\section{b) Zur Weiterentwicklung der Abkommen}

Der völkerrechtliche Charakter der Bilateralen Abkommen impliziert, dass sie grundsätzlich (formell) statische Verpflichtungen enthalten, so dass eine Modifikation des EU-Rechts, auf das in einem Abkommen Bezug genommen wird, grundsätzlich nicht „automatisch“ auch Eingang in das Abkommen findet. Allerdings sehen die Abkommen vor dem Hintergrund, dass ihre Zielsetzung in der Regel auch darin besteht, im Verhältnis zur Schweiz eine parallele Rechtslage wie innerhalb der EU zu gewährleisten, spezifische Bestimmungen für die Übernahme neuer legislativer Bestimmungen des EU-Rechts vor, wobei diese nur dann zum

\footnotetext{
15 Vgl. z.B. Anhang I Personenfreizügigkeitsabkommen, das teilweise wörtlich unionsrechtliche Bestimmungen übernimmt.

$16 \quad$ Vgl. schon NZZ v. 9.12.2008, 16.
} 
Zuge kommen, wenn der Verweis auf den unionsrechtlichen Besitzstand durch eine Bezugnahme auf Sekundärrechtsakte erfolgt.

Im Einzelnen können in den bestehenden Abkommen drei Kategorien von Übernahmemechanismen unterschieden werden:

- Erstens wird in zahlreichen Abkommen dem aus Vertretern der Vertragsparteien zusammengesetzten Gemischten Ausschuss die Kompetenz eingeräumt, die entsprechenden Anhänge des betreffenden Abkommens $\mathrm{zu}$ modifizieren. Da der Ausschuss einstimmig entscheidet, impliziert diese Variante (auf die insbesondere in Abkommen der „Bilateralen I“ zurückgegriffen wird), dass eine Anpassung der Abkommen an die Weiterentwicklung in der EU auch unterbleiben kann.

- Zweitens besteht teilweise eine grundsätzliche Pflicht der Schweiz zur Übernahme auch der Weiterentwicklungen des unionsrechtlichen Besitzstands, wobei es der Schweiz aber unbenommen bleibt, die Weiterentwicklungen unter Durchlaufen der ordentlichen (Gesetzgebungs-) Verfahren „umzusetzen““. Erfolgt jedoch keine Übernahme bestimmter Weiterentwicklungen, wird das Abkommen grundsätzlich - nach Ablauf einer bestimmten Frist - beendigt. Damit geht zwar auch dieses Modell nicht von einer „automatischen“ Übernahmepflicht der einschlägigen Weiterentwicklungen des Unionsrechts aus; im Falle des Scheiterns einer solchen Übernahme jedoch droht mit der Beendigung des Abkommens eine „Sanktion“. Vor diesem Hintergrund lässt dieser Mechanismus der Schweiz im Ergebnis relativ wenig bzw. kaum einen Spielraum, soweit die Übernahme der entsprechenden Weiterentwicklungen betroffen ist, da im Falle einer Ablehnung das gesamte Abkommen in Frage gestellt wird. Verankert wurde dieses Modell bislang in der Schengen-/ Dublin-Assoziierung.

- $\quad$ Ein ähnliches, wenn auch weniger weitgehendes Modell sieht zwar keine Beendigung des Abkommens im Falle fehlender Übernahme vor, ermöglicht aber der EU, im Falle des Ausbleibens der Übernahme von Weiterentwicklungen des Unionsrechts verhältnismäßige Ausgleichsmaßnahmen zu ergreifen, wobei der Gemischte Ausschuss ein Schiedsgericht anrufen kann, um die Verhältnismäßigkeit solcher Ausgleichsmaßnahmen zu überprüfen. Dieses Modell wurde bislang erst bei einem neueren Abkommen, nämlich dem Abkommen über die Erleichterung der Kontrollen und Formalitäten im Güterverkehr und über zollrechtliche Sicherheitsmaßnahmen (,24Stunden-Regel"s) ${ }^{17}$, herangezogen.

Vor dem Hintergrund des Bestrebens der EU, in Zukunft eine vollumfängliche Einbindung der Schweiz in den entsprechenden Teil des unionsrechtlichen Besitzstands sicherzustellen, wobei auch Weiterentwicklungen einzubeziehen sind, ist - auch nach den Aussagen von Vertretern der EU selbst ${ }^{18}$ - zu erwarten, dass die EU als Mindeststandard für zukünftige Abkommen ein

Fn. 3.

Vgl. schon die entsprechende Verlautbarung des Rates vom Dezember 2008, hierzu NZZ v. 9.12.2008, S. 16. 
Modell, das demjenigen der Schengen-Assoziierung nachgebildet ist, fordern wird. Es dürfte für die Schweiz schwierig sein, sich diesem Anliegen grundsätzlich zu verschließen, wenn auch punktuelle Ausnahmen nicht ausgeschlossen erscheinen. ${ }^{19}$

Nur am Rande sei in diesem Zusammenhang auch darauf hingewiesen, dass die Frage, ob ein bestimmter (neuer) EU-Rechtsakt eine Weiterentwicklung des von einem Abkommen erfassten unionsrechtlichen Besitzstands darstellt, mitunter schwierig zu eruieren und umstritten sein kann. So betrachtet die Union z.B. die neue Datenschutzgrundverordnung ${ }^{20}$ nicht als Weiterentwicklung der Schengen-/Dublin-Assoziierung, dies obwohl die mit dieser Verordnung aufgehobene Datenschutzrichtlinie ${ }^{21}$ Teil des von dieser Assoziierung erfassten EU-Besitzstands ist. ${ }^{22}$ Jedenfalls könnte der EuGH mit dieser Problematik befasst werden, da die Frage, ob ein bestimmter Rechtsakt eine Weiterentwicklung des Unionsbesitzstands im Sinne des jeweiligen Abkommens ist, eine Frage nach der Auslegung des betreffenden, von der Union abgeschlossenen Vertrages ist. ${ }^{23}$

Die Einbindung der Schweiz - über Bilaterale Abkommen - in Teile des unionsrechtlichen Besitzstands wirft die komplexe und hier nicht weiter zu vertiefende ${ }^{24}$ Frage auf, ob und inwieweit die entsprechenden Abkommensbestimmungen parallel zur Rechtslage im Unionsrecht auszulegen sind. Diese Frage ist - da es sich um völkerrechtliche Verträge handelt - ausgehend von völkerrechtlichen Auslegungsmethoden und jeweils gesondert für die verschiedenen Abkommen zu beantworten. Jedenfalls in den Fällen, in denen der Regelungszweck des Abkommens klar erkennen lässt, dass eine parallele Rechtslage angestrebt wird, spricht grundsätzlich Vieles für eine parallele Auslegung, wobei jedoch noch der zeitliche Bezugsrahmen zu präzisieren ist. Eine solche parallele Auslegung muss grundsätzlich auch und

19 Das etwas weniger weitgehende Modell, das dem neuen Abkommen über Zollerleichterungen und Zollsicherheit zugrundeliegt, wird von Vertretern der Europäischen Union insofern als „Sonderfall“ bezeichnet, als es um ein sehr technisches Abkommen gehe, das keinesfalls verallgemeinerungsfähig sei. Vgl. NZZ v. 7.7.2010.

20 VO 2016/679 zum Schutz natürlicher Personen bei der Verarbeitung personenbezogener Daten, zum freien Datenverkehr und zur Aufhebung der Richtlinie 95/46/EG (Datenschutz-Grundverordnung), AB1. 2016 L $119,1$.

21 RL 95/46 zum Schutz natürlicher Personen bei der Verarbeitung personenbezogener Daten und zum freien Datenverkehr, AB1. 1995 L 281, 31.

22 Vgl. zur Thematik Astrid Epiney/Markus Kern, Zu den Neuerungen im Datenschutzrecht der Europäischen Union, in: Astrid Epiney/Daniela Nüesch (Hrsg.), Die Revision des Datenschutzes in Europa und die Schweiz / La révision de la protection des données en Europe et la Suisse, Zürich 2016 (im Erscheinen). $\mathrm{Zu}$ den Kompetenzen des EuGH zur Auslegung völkerrechtlicher Verträge Astrid Epiney, in: Roland Bieber/Astrid Epiney/Marcel Haag, Die Europäische Union, 11. Aufl., 2015, § 9, Rn. 92, 95.

24 Vgl. ausführlich zur Auslegung der Bilateralen Abkommen, m.w.N., Epiney/Metz/Pirker, Parallelität der Rechtsentwicklung in der EU und in der Schweiz (Fn. 2), passim. 
gerade unter Berücksichtigung der Rechtsprechung des EuGH erfolgen, wie dies auch teilweise ausdrücklich in den Bilateralen Abkommen vorgesehen ist. ${ }^{25}$

Allerdings ist die Antwort auf die Frage nach einer parallelen Auslegung in Bezug auf die derzeit bestehenden Abkommen durchaus mit großen Unsicherheiten und Schwierigkeiten verbunden: Schon das Vorliegen eines Rückgriffs auf unionsrechtliche Begriffe kann streitig sein; weiter ist die Maßgeblichkeit der Weiterentwicklung der Auslegung auf EU-Ebene insbesondere durch die Rechtsprechung des EuGH - unklar. Insgesamt besteht daher in Bezug auf die Auslegung der Bilateralen Abkommen eine gewisse Rechtsunsicherheit, wenn auch in Bezug auf das Personenfreizügigkeitsabkommen, das in der gerichtlichen Praxis bislang am häufigsten relevant geworden ist, grundsätzlich eine parallele Auslegung zugrunde zu legen ist, wovon auch die Rechtsprechung im Ergebnis ausgeht, wobei hier zwischen derjenigen des Bundesgerichts ${ }^{26}$ und derjenigen des $\mathrm{EuGH}^{27}$ gewisse, allerdings jedenfalls inzwischen nicht mehr sehr ins Gewicht fallende Nuancen zu verzeichnen sind.

d) Rechtsschutzaspekte

In Bezug auf den Rechtsschutz im Rahmen Bilateraler Abkommen ist zwischen zwei Aspekten zu unterscheiden:

Geht es um den Rechtsschutz Einzelner, hat jede Vertragspartei - ggf. unter Beachtung abkommensrechtlicher Vorgaben - nach dem einschlägigen internen Recht Rechtsschutz zu gewähren. In den derzeit bestehenden Abkommen ist keine Möglichkeit schweizerischer Gerichte vorgesehen, dem EuGH Vorabentscheidungsfragen vorzulegen.

- Geht es um Streitigkeiten zwischen den Vertragsparteien, kommt bei den derzeit bestehenden Verträgen dem Gemischten Ausschuss eine zentrale Rolle zu. Gerichtliche Streitbeilegungsmechanismen sind grundsätzlich nicht vorgesehen und jedenfalls in Bezug auf abkommensrechtliche Vorgaben, die dem Unionsrecht entsprechen, auf der Grundlage der Rechtsprechung des EuGH ${ }^{28}$ ausgeschlossen.

Grundsätzlich stehen somit Anwendung und Auslegung der Abkommen in der EU einerseits und in der Schweiz andererseits in der Verantwortung der Vertragsparteien; ausdrückliche diesbezügliche Vorgaben enthalten die Abkommen nur ausnahmsweise. So geht die Schengenund Dublin-Assoziierung davon aus, dass eine einheitliche Anwendung und Auslegung des in

So etwa in Art. 16 Abs. 2 FZA.

S. insbesondere BGE 136 II 5.

S. insbesondere EuGH, Rs. C-70/09 (Hengartner), Slg. 2010, I-7233; inzwischen dürfte aber auch der EuGH von einer grundsätzlich parallelen Auslegung ausgehen, vgl. EuGH, Rs. C-506/10 (Graf), Slg. 2011, I-9345; EuGH, Rs. C-425/11 (Ettwein), ECLI:EU:C:2013:121; EuGH, Rs. C-656/11 (Vereinigtes Königreich/Rat), ECLI:EU:C:2014:97; EuGH, Rs. C-241/14 (Bukovansky), ECLI:EU:C:2015:766. Gutachten 1/91 (EWR I), Slg. 1991, I-6079; s. auch Gutachten 1/92 (EWR II), Slg. 1992, I-2821. 
das Abkommen integrierten unionsrechtlichen Besitzstands zu gewährleisten ist; kommt über eine entsprechende Frage im Gemischten Ausschuss keine Einigung zustande, wird das Beendigungsverfahren ausgelöst (vgl. Art. 8-10 Schengen-Assoziierung). Die übrigen Abkommen hingegen kennen - sieht man einmal von dem spezifische Charakteristika aufweisenden Luftverkehrsabkommen ab - keine entsprechenden Mechanismen.

\section{Aktuelle Herausforderungen für die Zukunft des „Bilateralen Weges“: zu den ,institutionellen Fragen“"}

Der skizzierte „Bilaterale Weg“" stellt für die Schweiz (aber wohl auch für die Union) insgesamt eine Erfolgsgeschichte dar, was auch für die mitunter umstrittene Personenfreizügigkeit gilt. ${ }^{29}$ Nichtsdestotrotz sieht er sich derzeit bedeutenden Herausforderungen und auch Infragestellungen ausgesetzt, die teilweise (aus Schweizer Sicht) „hausgemacht“ sind (wie insbesondere diverse Volksinitiativen und das Inkrafttreten von Verfassungsartikeln, die zumindest in einem gewissen Spannungsverhältnis zur Personenfreizügigkeit stehen), ihre Ursache aber auch teilweise in von Seiten der Union an die Schweiz herangetragenen Anliegen finden. Während der zuerst genannte Aspekt im vorliegenden Zusammenhang weniger interessiert und daher hier ausgespart werden soll, ${ }^{30}$ sind die von der Union formulierten Anliegen in Bezug auf die institutionelle Ausgestaltung der Bilateralen Abkommen auch vorliegend von großem Interesse.

So betont die Union seit mehreren Jahren, eine Weiterentwicklung der Bilateralen Abkommen und insbesondere der Abschluss neuer Abkommen, die einen Marktzugang und damit eine weitergehende Beteiligung am Binnenmarkt implizieren (woran die Schweiz ein Interesse haben kann, so etwa im Elektrizitäts- oder Dienstleistungsbereich), komme nur unter der Voraussetzung einer Einigung der Vertragsparteien über die Lösung der sog. institutionellen Fragen in Betracht. Im Zentrum des Interesses stehen dabei die Übernahme des unionsrechtlichen Besitzstandes und seine Auslegung, die Überwachung der Einhaltung der Abkommen in der und durch die Schweiz sowie die Streitbeilegung. ${ }^{31}$ Die Union geht dabei davon aus, dass grundsätzlich eine ,,automatische Übernahme“ neuen Unionsrechts im erfassten Bereich sowie eine parallele Auslegung der Begriffe und Rechtsakte, die aus dem Unionsrecht

$29 \quad$ Vgl. die Untersuchung der (positiven) Effekte der Freizügigkeit z.B. bei Patrik Schellenbauer, Wirtschaft und Arbeitsmarkt unter neuem Regime, in: Patrik Schellenbauer/Gerhard Schwarz (Hrsg.), Bilateralismus - was sonst? Eigenständigkeit trotz Abhängigkeit, Zürich 2015, 81 ff., m.w.N.

30 Vgl. insoweit Astrid Epiney, Freizügigkeit Schweiz - EU: status quo, Perspektiven und offene Fragen, in: Alberto Achermann u.a. (Hrsg.), Jahrbuch für Migrationsrecht 2015/2016, 2016, 3 ff.

31 Vgl. zur Problematik Epiney, in: Bilateralismus (Fn. 13), 25 (60 ff.). S. sodann z.B. Andreas Glaser/Lorenz Langer, Die Institutionalisierung der Bilateralen Verträge: Eine Herausforderung für die schweizerische Demokratie, SZIER 2013, 563 ff.; Joëlle de Sépibus, Ein institutionelles Dach für die Beziehungen zwischen der Schweiz und der Europäischen Union, in: Astrid Epiney/Stefan Diezig (Hrsg.), Schweizerisches Jahrbuch für Europarecht 2013/2014, 2014, 397 ff. 
übernommen werden, gewährleistet sein müsse. Weiter seien internationale Mechanismen der Überwachung und Streitbeilegung vorzusehen, wobei die Union offenbar den EWR als Leitbild vor Augen hat. ${ }^{32}$

Während sich die Schweiz und die Union in den bislang erfolgten Gesprächen - eigentliche Verhandlungen gibt es derzeit wegen der nach wie vor unklaren Umsetzung der sog. Masseneinwanderungsinitiative (Art. 121a BV) nicht - offenbar über die Grundsätze der Rechtsübernahme, der Auslegung sowie der Überwachung einigen konnten ${ }^{33}$ (letztlich wohl weitgehend auf der Linie der Anliegen der Union, was übrigens im Wesentlichen den bereits in der Schengen-/Dublin-Assoziierung verankerten Grundsätzen entsprechen dürfte, und bei der Auslegung legt - wie erwähnt - bereits die derzeitige Rechtsprechung einen Grundsatz der Parallelität zugrunde), bestehen offenbar im Zusammenhang mit der Streitbeilegung nach wie vor gewisse offene Fragen.

Der Bundesrat (die Regierung) plädiert in Bezug auf diesen Aspekt und damit die Frage der Gerichtsbarkeit für einen Einbezug des EuGH (eine Haltung, die durchaus auch auf Kritik gestoßen ist): ${ }^{34}$ Im Falle der Uneinigkeit beider Parteien im Gemischten Ausschuss über die rechtliche Tragweite bzw. die Auslegung einzelner Bestimmungen der Abkommen kann eine der Parteien beim EuGH ein Gutachten anfordern, das sich auf die Auslegung der entsprechenden Bestimmungen der Bilateralen Abkommen (wobei nur solche Bestimmungen, die den Binnenmarktacquis aus dem EU-Recht in die Abkommen übernehmen, betroffen sein sollen) bezieht. Das Gutachten des Gerichtshofs implizierte eine autoritative Auslegung der entsprechenden Abkommensbestimmungen für beide Parteien, und im Gemischten Ausschuss würden die Folgen des Gutachtens erörtert und bestimmt. Allerdings bliebe es der Schweiz unbenommen, die durch den EuGH vorgenommene Auslegung gleichwohl nicht zu akzeptieren; dies führte jedoch nicht dazu, dass die Auslegung des Gerichtshofs als solche nicht mehr ,autoritativ“ wäre, sondern gäbe der EU die Möglichkeit, Gegenmaßnahmen zu ergreifen bzw. gar das Abkommen zu suspendieren. Die Verhältnismäßigkeit dieser Maßnahmen könnte durch ein Schiedsgericht überprüft werden. Diese an sich elegante Lösung, die nach der hier vertretenen (aber durchaus umstrittenen) Ansicht ${ }^{35}$ viele Vorteile aufweist, muss aber noch im Einzelnen präzisiert werden, wobei aus der Sicht der Union insbesondere sicherzustellen ist, dass die Urteile des EuGH - wie sich aus seiner Rechtsprechung ergibt - verbindlich sein müssen, ${ }^{36}$ so dass die Union möglicherweise auf einer Art Beendigungsmechanismus im Falle einer „Nichtbeachtung“ der Urteile des Gerichtshofs bestehen könnte, was sich nicht in jeder Beziehung mit den Vorstellungen des Bundesrates decken könnte und im Übrigen auch die

\footnotetext{
$32 \quad$ Vgl. NZZ v. 21.12.2012.

33 Vgl. hierzu etwa NZZ vom 21.11.2012. S. den aktuellen Stand jeweils auf www.admin.ch.

34 Vgl. z.B. Christa Tobler, Die Erneuerung des bilateralen Wegs: eine wachsende Annäherung an den EWR in den zur Diskussion gestellten Modellen, Jusletter, v. 3.6.2013. S. aber auch die Zustimmung bei Epiney, in: Bilateralismus (Fn. 13), 25 (65 ff.).

35 S. die Nachweise in Fn. 34.

36 Vgl. EuGH, Gutachten 1/91 (EWR I), Slg. 1991, I-6079; EuGH, Gutachten 1/09 (Europäisches Patentgericht), Slg. 2011, I-1137; s. auch Gutachten 1/92 (EWR II), Slg. 1992, I-2821.
} 
Frage nach der politischen Akzeptanz einer solchen Lösung in der Schweiz aufwürfe. Nach der hier vertretenen Ansicht ist der vom Bundesrat vertretene Ansatz aber schon deshalb mit den Verträgen vereinbar, weil die Auslegung als solche eben gerade verbindlich bleiben soll, so dass der vom Bundesrat angedachte Mechanismus letztlich eine Art vertraglich vorgesehene Möglichkeit der Nichteinhaltung eines Vertrages impliziert.

Auch wenn diese Fragen einer Lösung zugeführt werden können, bleiben noch zahlreiche weitere Fragen offen: So ist es beim derzeitigen Stand der Dinge unklar, ob und ggf. inwieweit ein solcher neuer institutioneller Rahmen auch auf die bereits bestehenden Abkommen Anwendung fände. Eine Ausdehnung des neuen institutionellen Rahmens z.B. auf das Freizügigkeitsabkommen würfe aber komplexe Fragen auf. So müsste geklärt werden, auf welche Weise dies beim Freizügigkeitsabkommen - das ja ein gemischtes Abkommen ist, welches auf Seiten der Union neben der EU auch die Mitgliedstaaten als Vertragsparteien umfasst - eine allfällige Ausdehnung völkerrechtlich verankert werden soll, insbesondere, ob die Ratifizierung aller EU-Mitgliedstaaten erforderlich wäre. Aber auch die rechtstechnische Sicherstellung einer (weitgehenden) Homogenität der Rechtsentwicklung stieße bei zahlreichen Abkommen auf erhebliche Schwierigkeiten, dies in den Konstellationen, in denen die Übernahme des Unionsrechts nicht durch Verweise auf Sekundärrechtsakte, sondern durch eine entsprechende Formulierung der Abkommenstexte selbst erfolgt, müssten diesfalls doch die Abkommen entsprechend modifiziert werden, was auch Fragen ihres materiellen Geltungsbereichs aufwürfe, erfolgt doch mitunter nur eine teilweise Anlehnung an EU-Recht. Im Übrigen gilt auch bezüglich der institutionellen Fragen, dass das Ergebnis der BrexitAbstimmung in Großbritannien die Verhandlungsposition der Schweiz eher geschwächt haben dürfte, wird doch die Union jede diskutierte und in Betracht kommende Lösung auch unter dem Gesichtspunkt analysieren, ob sie insofern verallgemeinerungsfähig ist, dass sie auch im Verhältnis zu Großbritannien zum Zuge kommen könnte. Insofern stehen also die bevorstehenden Verhandlungen der EU mit Großbritannien über die zukünftige Gestaltung des gegenseitigen Verhältnisses in einer Wechselbeziehung mit der Frage nach der Weiterentwicklung der Beziehungen Schweiz - EU.

\section{Fazit: Eckwerte „Bilateraler Abkommen Großbritannien - EU“}

Vor diesem Hintergrund sei im Folgenden danach gefragt, welche Folgerungen sich aus den Beziehungen Schweiz - EU für die Perspektiven der Gestaltung des Verhältnisses von Großbritannien zur Union ziehen lassen, dies soweit grundsätzlich die Idee verfolgt werden sollte, hier eine ähnliche Architektur vorzusehen. In der Tat lassen sowohl die Ausgestaltung der bestehenden Bilateralen Abkommen der Schweiz mit der Union als auch die derzeit diskutierten offenen Fragen bzw. die diesbezüglichen Stellungnahmen der Union Rückschlüsse 
darauf zu, innerhalb welcher „Leitplanken“ sich die abzuschließenden Abkommen mit Großbritannien (für den Fall der Zugrundelegung eines „Bilateralen Ansatzes“) voraussichtlich bewegen werden. Dies beruht im Wesentlichen auf der Überlegung, dass die Verhandlungsposition der Union - die sich im Übrigen in den vergangenen Jahren durchaus weiterentwickelt hat, so dass z.B. anzunehmen ist, dass die 1999 unterzeichneten „Bilateralen I“ heute in dieser Form nicht (mehr) abgeschlossen werden könnten - maßgeblich davon geprägt ist, Drittstaaten insgesamt keine vorteilhaftere Position einzuräumen als den Mitgliedstaaten, eine Haltung, die sich im Zuge des Brexit wohl eher noch verstärken wird. Dieser Ansatz impliziert letztlich, dass in all denjenigen Bereichen (und hier spielt selbstredend der Binnenmarkt eine besonders wichtige Rolle, wobei man aber auch auf die Teilnahme an diversen Programmen, wie etwa den Forschungsprogrammen, hinweisen kann), in denen der Drittstaat ein Interesse an einer Teilhabe an einem bestimmten Teil des unionsrechtlichen Besitzstands hat, diese Teilnahme letztlich auch eine Übernahme des entsprechenden unionsrechtlichen Besitzstands sowie gewisser institutioneller Grundsätze mit sich bringt.

Auch wenn hier jeweils noch zahlreiche Einzelfragen zu klären sind, vermag die Ausgestaltung der Beziehungen Schweiz - EU und ihre Entwicklung die Konkretisierung dieses in dieser Form noch sehr allgemein formulierten Anliegens zu illustrieren. Diese lassen - soweit es um Bereiche geht, welche die Einbindung eines Drittstaats in einen Teil des unionsrechtlichen Besitzstands zum Gegenstand haben, dies letztlich mit dem Ziel, im Verhältnis zu dem Drittstaat eine (im Wesentlichen) parallele Rechtslage wie zwischen EU-Mitgliedstaaten zu gewährleisten (wie dies typischerweise bei der Teilnahme am Binnenmarkt der Fall ist) folgende Eckpunkte erkennen, die im Grundsatz aus Sicht der Union wohl kaum verhandelbar sind:

Reichweite der Übernahme des unionsrechtlichen Besitzstands: Der relevante unionsrechtliche Besitzstand wäre zu übernehmen, wobei diese Übernahme in Zukunft wohl in erster Linie durch einen Verweis auf die einschlägigen EU-Rechtsakte erfolgte. Auch die Weiterentwicklungen des vom Abkommen erfassten unionsrechtlichen Besitzstands wären wohl grundsätzlich zu übernehmen. Im Gegenzug würde wohl eine gewisse (konsultative) Beteiligung am Erlass der einschlägigen Rechtsakte verankert werden können.

Regelmäßig problematisch wird in diesem Zusammenhang die genaue Definition des zu übernehmenden Besitzstands sein, wobei sich diese Frage sowohl in Bezug auf den bestehenden Besitzstand als auch auf seine Weiterentwicklungen bezieht. Die Union neigt insgesamt eher zu einer weiten Auslegung des zu übernehmenden Besitzstands, dies letztlich mit dem Argument, Aufteilungen seien aufgrund des Gesamtzusammenhangs schwierig. ${ }^{37}$

37 So formulierte die EU im Rahmen der Verhandlungen über ein Elektrizitätsabkommen mit der Schweiz offenbar das Anliegen, es seien auch eine Reihe umweltrechtlicher Aspekte zu regeln, so dass die Schweiz nicht nur den „Elektrizitätsacquis“, sondern auch Rechtsakte in den Bereichen Umweltverträglichkeit, 
In Bezug auf Großbritannien könnte sich hier konkret die Frage stellen, ob die Personenfreizügigkeit ohne eine Übernahme der Unionsbürgerrichtlinie in einem Abkommen verankert werden könnte. ${ }^{38}$ Weiter könnte die Union gewisse sonstige Binnenmarktabkommen von einem Abkommen über die Personenfreizügigkeit abhängig machen. Nichtsdestotrotz dürfte bei der Festlegung der Reichweite des zu übernehmenden Besitzstands wohl ein gewisser Verhandlungsspielraum bestehen.

- $\quad$ Auslegung und Anwendung: Entsprechend der Rechtslage in der Schengen-/DublinAssoziierung Schweiz - EU sowie in Anknüpfung an die entsprechenden Anliegen im Zusammenhang mit den ,,institutionellen Fragen“ wäre grundsätzlich in Großbritannien eine parallele Auslegung und Anwendung der Abkommen wie in der EU sicherzustellen. Dies impliziert eine Pflicht zur Beachtung der einschlägigen EuGHRechtsprechung sowohl zum Zeitpunkt der Unterzeichnung des Abkommens als auch letztlich ihrer Weiterentwicklung. Die Sicherstellung der effektiven Beachtung dieses Grundsatzes könnte im Wesentlichen insbesondere durch die Einrichtung eines Beendigungsmechanismus - ebenfalls entsprechend demjenigen, der in der Schengen/Dublin-Assoziierung verankert ist - erfolgen.

Die Sicherstellung einer solchermaßen parallelen Auslegung ist im Übrigen eng mit dem Übernahmemechanismus verbunden: Eine gewisse Schwierigkeit jedenfalls bei den derzeit mit der Schweiz abgeschlossenen Bilateralen Abkommen besteht nämlich darin, dass die genaue Reichweite der Bezugnahme auf unionsrechtliche Begriffe und Konzepte nicht immer ganz klar ist, was mitunter auch auf der manchmal fehlenden Übernahme von Weiterentwicklungen des Unionsrechts beruht. M.a.W. drängt es sich auch und gerade im Hinblick auf eine möglichst weitgehende Rechtsklarheit und damit Rechtssicherheit auf, soweit wie möglich die Bezugnahme auf unionsrechtliche Begriffe hinreichend deutlich zu verankern (soweit nicht von vornherein auf Sekundärrecht Bezug genommen wird).

Überwachung und Streitbeilegung zwischen den Vertragsparteien: Da die EUMitgliedstaaten hier supranationalen Mechanismen unterliegen, müssten für Drittstaaten zumindest internationale Mechanismen zum Zuge kommen, so dass hier nicht die für das Völkerrecht typische Zweisäulenstruktur Anwendung finden könnte, übrigens eine Kernforderung der Union gegenüber der Schweiz im Zusammenhang mit

Industrieemissionen sowie Naturschutz übernehmen würde bzw. zumindest eine gleichwertige und damit im Wesentlichen unionsrechtskompatible Rechtslage in der Schweiz bestehen müsste. Vgl. hierzu nur Astrid Epiney/David Furger/Jennifer Heuck, „Umweltplanungsrecht“ in der Europäischen Union und Implikationen für das schweizerische Recht, 2011, $2 \mathrm{f}$.

38 Dass die Schweiz diese Richtlinie nicht übernommen hat, beruht allein darauf, dass sie einige Jahre nach dem Abschluss des Personenfreizügigkeitsabkommens erlassen wurde; einer Anfrage der Union, sie in das Abkommen zu integrieren, erteilte die Schweiz eine Absage. Vgl. hierzu, m.w.N., Astrid Epiney/Robert Mosters, Die Rechtsprechung des EuGH zur Personenfreizügigkeit im Jahre 2015 und ihre Implikationen für das Freizügigkeitsabkommen Schweiz - EU, in: Astrid Epiney/Markus Kern/Lena Hehemann (Hrsg.), Schweizerisches Jahrbuch für Europarecht / Annuaire Suisse de droit européen 2015/2016, 2016,63 (67 f.). 
den ,,institutionellen Fragen“. Denkbar sind hier verschiedene Ansätze, wobei - auf der Grundlage der Rechtsprechung des EuGH, der insbesondere im Zusammenhang mit dem EWR festhielt, die „Unterwerfung“ der Union (im Rahmen eines völkerrechtlichen Vertrages) unter eine internationale Gerichtsbarkeit beeinträchtige jedenfalls in den Fällen, in denen dieses Gremium verbindlich über die Auslegung von Bestimmungen, welche dem Unionsrecht entlehnt sind, zu entscheiden habe, die Autonomie des EuGH bzw. die durch die Verträge vorgesehenen Gerichtsbarkeit ${ }^{39}$ - im Wesentlichen entweder eine internationale Zweisäulenstruktur nach dem Vorbild des EWR bzw. des EFTA-Gerichtshofs (der verbindlich nur für die EWR-Staaten entscheidet) oder aber ein Einbezug des EuGH in dem Sinn, dass dieser auch für die Drittstaaten verbindliche Urteile fällt bzw. Gutachten erstellt, in Betracht kommen. Je nach gewählter Lösung käme für die Überwachung der EFTA-Überwachungsbehörde oder aber der Kommission eine gewisse Rolle zu. Die Einzelheiten wären zu verhandeln.

- Rechtsschutz Einzelner: Denkbar wäre die Einführung eines dem Vorabentscheidungsverfahren nachgebildeten Vorlageverfahrens (vor dem EFTAGerichtshof oder dem EuGH), was aber die Verbindlichkeit der daraufhin ergangenen Urteile implizierte. Angesichts der bisherigen Erfahrungen scheint ein solcher Mechanismus aber aus Sicht der Union nicht zu den Kernelementen der institutionellen Fragen zu gehören.

- $\quad$ Schiedsgericht: Die Einrichtung eines Schiedsgerichts bei Streitigkeiten zwischen den Vertragsparteien, die nicht dem Unionsrecht nachgebildete Abkommensbestimmungen betreffen (z.B. die Verhältnismäßigkeit von in Abkommen vorgesehenen Gegenmaßnahmen), erscheint möglich.

Beendigung des Abkommens: Seit spätestens 2008 insistiert die Union auf der Notwendigkeit einer Art Garantie einer homogenen Rechtsentwicklung in Bereichen bzw. bei Abkommen, die eine Teilnahme an Teilen des unionsrechtlichen Besitzstands vorsehen. Dies impliziert, dass im Falle einer Ablehnung der Übernahme einer Weiterentwicklung des Unionsrechts durch den Drittstaat das Abkommen nach Ablauf einer gewissen Frist automatisch beendet würde, es sei denn, der Gemischte Ausschuss entscheide etwas anderes, ein Modell, dass derzeit in der Schengen-/DublinAssoziierung mit der Schweiz verankert ist und von der Union als „Modell“ für zukünftige Abkommen angesehen wird.

Deutlich wird damit, dass die Option „Bilaterale Abkommen“ als Modell für die zukünftige Gestaltung der Beziehungen Großbritanniens zur Union durchaus eine ins Gewicht fallende Reduktion des Handlungsspielraums des (neuen) Drittstaats mit sich brächte. Dem stünden freilich auch beachtliche Vorteile gegenüber, namentlich eine Beteiligung an den

39 EuGH, Gutachten 1/91 (EWR I), Slg. 1991, I-6079; EuGH, Gutachten 1/09 (Europäisches Patentgericht), Slg. 2011, I-1137; s. auch Gutachten 1/92 (EWR II), Slg. 1992, I-2821. 
entsprechenden Teilen des unionsrechtlichen Besitzstands, was insbesondere für eine Teilnahme Großbritanniens am Binnenmarkt oder (bedeutenden) Teilen desselben ins Gewicht fällt. In der Tat dürfte es nicht realistisch sein anzunehmen, die Union würde einem (zukünftigen) Drittstaat die Teilnahme am Binnenmarkt - woran Großbritannien offenbar ein sehr großes Interesse hat - ermöglichen, ohne gleichzeitig zumindest grundsätzlich auf den skizzierten Eckpunkten zu bestehen.

Dies ändert nichts daran, dass es letztlich eine politische Entscheidung ist, für welche Art von Beziehungen zur Union sich Großbritannien (und die Union, ist doch ein Konsens notwendig) letztlich entscheiden wird. Denn freilich kommen auch andere Optionen in Betracht, so z.B. der EWR (der freilich in Bezug auf die Einschränkung des Handlungsspielraums noch über eine „Bilaterale Lösung“ hinausgehen dürfte), ein „reines“ Freihandelsabkommen oder ein „modernes“ Freihandelsabkommen (nach dem Vorbild der TTIP), eine Zollunion (ähnlich wie die Beziehungen zur Türkei) oder ein allgemeines „Zurückfallen“ auf das Welthandelsrecht. Soweit ersichtlich, ermöglichen aber diese Optionen (abgesehen vom EWR) gerade keine Teilnahme am Binnenmarkt, und im Übrigen eröffnete ein „Bilateraler Weg“ doch im Vergleich zum EWR durchaus gewisse Handlungsspielräume, die es erlauben, auf „Sonderwünsche“ einzugehen, dies trotz aller erkennbarer grundsätzlicher Eckpunkte. Insofern könnte es für Großbritannien zumindest überlegenswert sein, diesen Weg möglichst rasch prioritär zu verfolgen; es ist nämlich keineswegs sicher, dass die Union einer solchen, insgesamt dann eben doch eher komplexen Konstruktion auch dann noch positiv gegenüber steht, wenn mit der Schweiz keine Fortschritte zu verzeichnen sind, ist doch nicht zu vergessen, dass für die Teilnahme am Binnenmarkt an sich der EWR maßgeschneidert ist. 